\title{
Lattice QCD at finite temperature: a status report *
}

\author{
F. Karsch \\ CERN, CH-1211 Geneva 23, Switzerland
}

\begin{abstract}
We analyze the status of numerical simulation of QCD at finite temperature. Emphasis is put on quantitative predictions emerging from lattice calculations that may be tested in heavy ion experiments. Recent results on the chiral phase transition, thermodynamics of the quark gluon plasma phase and various screening lengths are discussed.
\end{abstract}

\section{Introduction}

At the first Quark-Matter conference in 1982 [1], results from Monte Carlo simulations of QCD, which we now would call first exploratory studies, have been discussed enthusiastically. They revealed the possibility that the creation of a quark-gluon plasma in heavy ion collisions is within reach of present days accelerator technology. Since then we learned a lot about the inherent problems of lattice simulations. Finite size effects, critical slowing down and violations of asymptotic scaling forced us to investigate larger lattices. However, also simulation techniques have improved steadily and efficient algorithms for the incorporation of light dynamical fermions have been developed. Thus much progress has been made since then.

During this year Quark-Matter Conference first experimental results from the CERN heavy-ion experiments will be presented [2]. This is the first step in an extensive search for a new phase of strongly interacting matter predicted by QCD. From lattice gauge theory we expect to obtain quantitative predictions for the critical parameters of the phase transition to the quark-gluon (QG) plasma as well as a deeper understanding of the non-perturbative aspects of this new phase. Unfortunately, lattice simulations are still

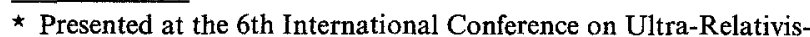
tic Nucleus-Nucleus Collisions - Quark Matter 1987, 24-28 August 1987, Nordkirchen, Federal Republic of Germany
}

restricted to the finite temperature $(T)$, vanishing chemical potential $(\mu)$ sector of the QCD. The problems in simulating systems with non-zero baryon number density have not yet been overcome [3]. However, for $T \neq 0, \mu=0$ we have now accurate results for the critical parameters of the phase transition in the pure gauge sector [4-6]. Simulations with dynamical fermions have reached a stage where large scale projects lead to first quantitative results for QCD with "nearly" massless quarks [7-12]. These simulations give strong evidence for the existence of a first order chiral phase transition in QCD. Moreover, the critical temperature shows similar behaviour as in the pure gauge sector. This leads us to hope that the presence of dynamical fermions will not alter completely the picture gained from the analysis of the pure gauge sector.

From an analysis of global thermodynamic observables like energy density and pressure, we hope to get insight into the basic properties of the QCD plasma phase. These quantities are important to judge the initial energy density needed in a heavy ion collision to create a QG plasma in a small space volume. Moreover, lattice simulations allow to study non-perturbative aspects of the high temperature plasma phase through the analysis of various screening lengths [13-16]. In particular, a quantitative understanding of Debye screening in the quark-gluon plasma the discussion of heavy quark bound state suppression as a signal for plasma formation [17].

In the following we will discuss the present status of the quantitative analysis of these observables. In the next section we review results for the critical temperature both in the pure gauge sector as well as in the presence of dynamical fermions. In Sect. 3, we will discuss perturbative, as well as non-perturbative, aspects of the equation of state in the plasma phase. Section 4 is devoted to a discussion of screening lengths in the quark-gluon plasma. Our conclusions are given in Sect. 5 . 


\section{QCD phase diagram at finite temperature}

The existence of a phase transition in QCD from a low temperature confining phase with spontaneously broken chiral symmetry to a high temperature deconfined, chiral symmetric phase is one of the fundamental predictions of QCD. In fact, the existence of such a phase transition has been proved rigorously on the lattice $[18,19]$. The bounds on the transition temperature, however, are weak and an infinite temperature in the continuum limit is not excluded by these proofs. A quantitative determination of the transition temperature has to rely on Monte Carlo simulations. To this extent, one analyzes the symmetry properties of the QCD action. The spontaneous breaking/restoration of certain symmetries at finite temperature signals the presence of a phase transition. The QCD partition function depends on the lattice size in space $\left(N_{\sigma}\right)$ and time $\left(N_{\tau}\right)$ direction, the number of quark species $\left(n_{f}\right)$, their masses in units of the lattice spacing ( $m a$ ) as well as on the bare coupling $\mathrm{g}^{2}$ :

$Z\left(N_{\sigma}, N_{\tau}, n_{f}, m a, g^{2}\right)=\int \prod_{x, \mu} d U_{x, \mu} e^{-S_{\text {efr }}}$

with the effective action given by

$$
\begin{aligned}
S_{\text {eff }}= & \frac{6}{g^{2}} \sum_{\text {plaquettes }}\left(1-\frac{1}{3} \operatorname{Re} \operatorname{Tr} U_{x, \mu} U_{x+\mu, \nu} U_{x+v, \mu}^{+} U_{x, \nu}^{+}\right) \\
& -\frac{n_{f}}{4} \operatorname{Tr} \ln Q_{f}
\end{aligned}
$$

Here $Q_{f}$ denotes the fermion matrix which in the case of staggered fermions is given by

$$
\begin{aligned}
Q_{f}^{x y}= & m a \delta_{x y}+\frac{1}{2} \sum_{v=0}^{3}\left[\delta_{y, x+v} U_{x, v}-\delta_{y, x-v} U_{x, v}^{+}\right] \\
& \rightleftharpoons(-1)^{x_{0}+\ldots+x_{v-1}} .
\end{aligned}
$$

$U_{x, \mu} \in S U(3)$ denotes gauge field variables which are defined on links $(x, \mu)$ of a four-dimensional lattice of size $N_{\sigma}^{3} \times N_{\tau}$.

The phase structure of QCD at finite temperature and order of the phase transitions has by now been studied for various number of flavours and a wide range of quark masses. Let us first discuss the pure gauge sector $\left(n_{f}=0\right.$ or $\left.m a \rightarrow \infty\right)$ which is best analyzed. The phase transition is characterized by a spontaneous breaking of the global $Z(N)$ centre symmetry of the pure $S U(N)$ gauge action. As an order parameter for this deconfinement phase transition serves the Polyakov loop operator

$$
\left\langle\operatorname{Tr} L_{\mathbf{x}}\right\rangle=\left\langle\operatorname{Tr} \prod_{x_{0}=1}^{N_{\tau}} U_{\left(x_{0}, \mathbf{x}\right), 0}\right\rangle \begin{cases}=0, & T \leqq T_{c} \\ >0, & T<T_{c}\end{cases}
$$

Already first simulations for $S U(3)$ indicated that the deconfinement phase transition is first order [20]. However, the critical temperature turned out not to scale according to the asymptotic scaling law

$T_{c} / A_{L}=\frac{1}{N_{\tau}}\left(\frac{8 \pi^{2}}{33} \frac{6}{g_{c}^{2}}\right)^{-51 / 121} e^{\frac{4 \pi^{2}}{33} \frac{6}{g_{c}^{2}}}$.

This was not unexpected as Monte Carlo renormalization group studies also indicated that couplings $6 / g^{2} \geq 6.1$ are needed in order to see the above asymptotic scaling behaviour [21]. Indeed constant critical temperatures have been found only for $N_{\tau} \geqq 10$ where the critical couplings are larger than $6 / g^{2}=6.0[4-6]$. For these large temporal lattices a direct observation of co-existing states is difficult. As the spatial extent of these lattices is only about twice as large, i.e., $N_{\sigma}$ $=2 N_{\tau}$, metastable states are observable in a whole region of $\beta$-values. This makes the precise location of the critical coupling difficult and to some extent dependent on the criterion used to define $\beta_{c}$. However, simulations of different groups seem to agree quite well [4-6]. These results are presented in Fig. 1. From this we get for the critical temperature in the pure gauge sector

$T_{c} / \Lambda_{\overline{M S}}=1.78 \pm 0.03$

where we have used $\Lambda_{\overline{M S}} / \Lambda_{L}=28.82$ to convert the lattice scale parameter to the $\overline{M S}$-scheme. This allows a simpler comparison with dynamical fermion calculations, as the scale parameter is less sensitive to the number of flavours in this scheme.

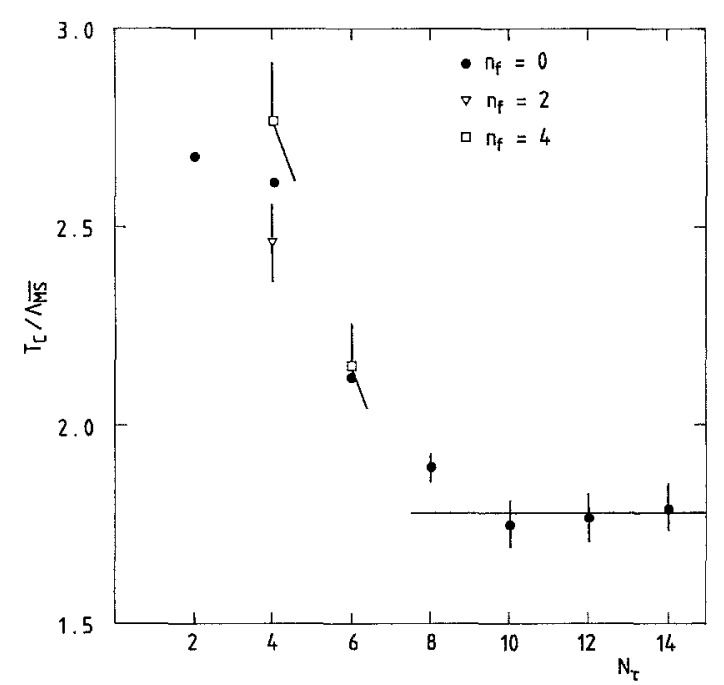

Fig. 1. Critical temperature in units of $A_{M S}$ versus temporal lattice size $N_{\tau}$. Shown are data for $S U(3)$ pure gauge theory, $n_{f}=0$, (dots) [4-6] and $S U(3)$ with dynamical fermions, $n_{f}=0$. The $n_{f}=2$ data (triangle) are taken from $[10,11]$ and those for $n_{f}=4$ (squares) are from $[8,9]$ 
To extract $T_{c}$ in units of $\mathrm{MeV}$ from (2.8), we have to compare with measurements of other physical observables in the asymptotic scaling regime. Using recent data for the string tension [22] and hadron masses [23] we find

$T_{c} / \sqrt{6}=0.58 \pm 0.04 \rightarrow T_{c}=(254 \pm 18) \mathrm{MeV}$

$T_{c} / m_{\rho}=0.30_{-0.02}^{+0.05} \rightarrow T_{c}=\left(231 \pm{ }_{15}^{38}\right) \mathrm{MeV}$

$T_{c} / m_{N}=0.21 \pm 0.02 \rightarrow T_{c}=(197 \pm 20) \mathrm{MeV}$

where we have used $\sigma=0.192 \mathrm{GeV}^{2}[24]$ for the string tension. The difference obtained for $T_{c}$ from measurements of the rho-meson and nucleon mass reflects of course that their mass ratio is still not well determined from quenched Monte Carlo simulations.

It has been expected that in the presence of dynamical fermions the strong first order deconfinement transition of the pure gauge sector gets weakened and would eventually disappear [25]. This is indeed what has been observed in an intermediate quark mass regime for a limited regime of flavours [26]. However, this observation is strongly dependent on the number of flavours and quark masses. For $n_{f} \geqq 8$ only first order transitions have been observed for all quark masses studied so far [27].

The crucial question, however, is what happens in the chiral limit, $m a \rightarrow 0$. Universality arguments favour first order transitions for $n_{f} \geqq 3$ [28]. For $n_{f}$ $=2$ the situation is unclear and the order of the transition depends strongly on the temperature dependence of the axial anomaly [28].

First indications for a first order chiral transition have been found by R. Gupta et al. [7]. They used an exact fermion algorithm on a $4^{4}$ lattice. During the last year, it became possible to perform reliable Monte Carlo simulations with light fermions on larger lattices which gave evidence for a first order chiral transition for different numbers of flavours [812]. In Fig. 2, we show the behaviour of the chiral order parameter $\langle\bar{\chi} \chi\rangle$ as well as the Polyakov loop $\langle L\rangle$ as a function of $\beta=6 / \mathrm{g}^{2}$ for the $n_{f}=4$ theory. The discontinuity at $\beta_{c}=4.96 \pm 0.03$ is clearly visible. It seems, however, that quark masses smaller than $m / T=0.15$ are necessary to observe this first order singularity. Calculations for other values of $n_{f}$ seem to indicate that the first order transition weakens with decreasing number of flavours. Even smaller quark masses are needed to find signals for a first order singularity. Indeed the order of the transition is controversal for $n_{f}=2[10,11]$. Although a recent detailed analysis seems to favour a first order transition also for $n_{f}=2$ [29]. At present the location of the critical quark mass below which a first order chiral transition is observed is very uncertain. There are

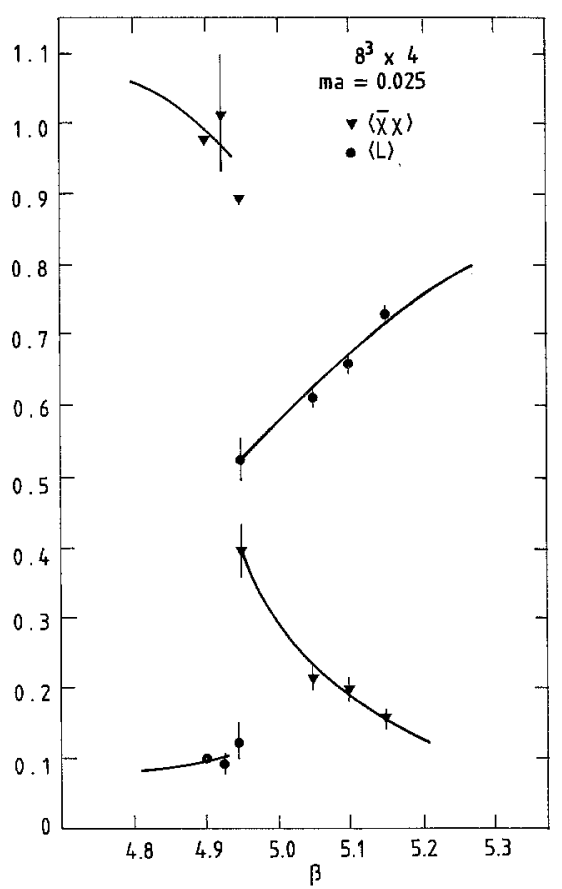

Fig. 2. First order discontinuity in the chiral order parameter $\langle\bar{\chi} \chi\rangle$ (triangles) and the Polyakov loop $\langle L\rangle$ (dots). Shown are $\langle\bar{\chi} \chi\rangle$ and $\langle L\rangle$ versus $\beta$ for the $S U(3), n_{f}=4$ theory (Fig. from [9])

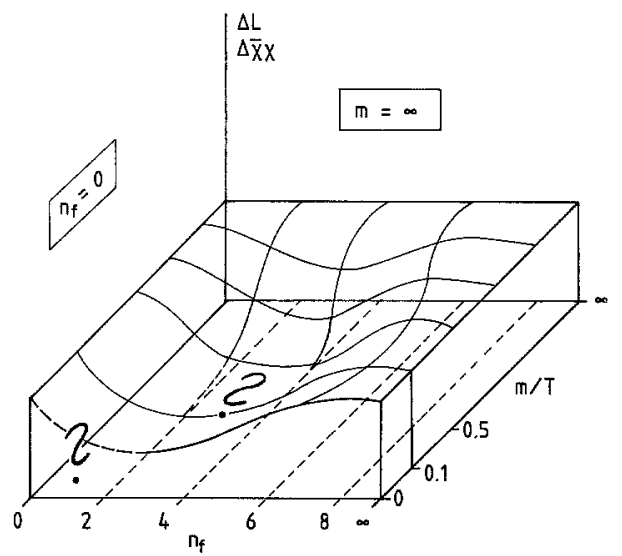

Fig. 3. Schematic view of the $S U(3)$ finite temperature phase diagram in the $m / T-n_{f}$ plane. Shown is the gap in various observables which signal the presence of a first order transition. Question marks indicate the regions of present numerical and/or theoretical uncertainties

large discrepancies between results of different groups using different algorithms. In particular the Langevin algorithm seems to lead to larger critical quark mass. At present we also cannot rule out the possibility that the QCD phase transition is first order for all quark masses.

The phase diagram in the $m / T, n_{f}$ plane that emerges from these calculations is shown in Fig. 3. Notice that there seems to be a small region in this plane where the transition to the high temperature 
Table 1. Comparison of critical temperatures obtained on small lattices for various number of flavours. The numbers are taken from the references given in brackets

\begin{tabular}{llll}
\hline$N_{\tau}$ & \multicolumn{1}{l}{$T_{c} / \Lambda_{\text {KS }}$} & \\
\cline { 2 - 4 } & $n_{f}=0$ & $n_{f}=2$ & $n_{f}=4$ \\
\hline 4 & $2.61 \pm 0.01[4,5]$ & $2.46 \pm 0.1[10]$ & $2.77 \pm 0.15[9]$ \\
6 & $2.12 \pm 0.01[4,5]$ & & $2.6 \pm 0.1[11]$ \\
\hline
\end{tabular}

phase is continuous. It remains to be seen whether this region is connected to the $m / T=0$ axis for small number of flavours, or whether it is present at all.

Compared to the pure gauge sector the chiral transition temperature has been determined only on rather small lattices. Clearly, the asymptotic scaling regime has not been reached up to now in simultaneous with dynamical fermions. Indeed $T_{c h} / \Lambda_{M S}$ shows similar scaling violations as the deconfinement temperature on small lattices. This becomes clear from Table 1 where we compare results for $n_{f}=0$, 2 and 4 on small lattices. These numbers are also plotted in Fig. 1. Using results from hadron mass calculations with dynamical fermions [30], the chiral transition temperature can be converted into physical units. This gives $T_{c h} \simeq 130-170 \mathrm{MeV}[10,11]$. However, given the present status of hadron mass calculations and the additional extrapolations involved*, this number should be taken with caution. Certainly much better numbers will be available in the near future where we also can expect to see first results for a realistic mass spectrum of two nearly mass-less up and down quarks and a strange quark of intermediate mass [31].

\section{Equation of state of the quark-gluon plasma}

Besides knowing the critical temperature $T_{c}$ for a phase transition to a quark-gluon plasma, an even more important quantity is the energy density required to reach this new phase of QCD. The early calculations for $S U(2)$ [32] and $S U(3)$ [20, 23] gauge theories showed that the energy density was close to the ideal gas value

$\varepsilon_{S B} / T^{4}=\frac{\pi^{2}}{15}\left(N^{2}-1\right)$

already immediately above $T_{c}$. On small lattices rather large finite size corrections have to be taken into ac-

\footnotetext{
* As the hadron mass calculations have not been performed directly at the critical coupling $6 / g_{c}^{2}$, the results have to be extrapolated to this value using the asymptotic renormalization group equation and assumptions about the amount of scaling violation in this coupling region.
}

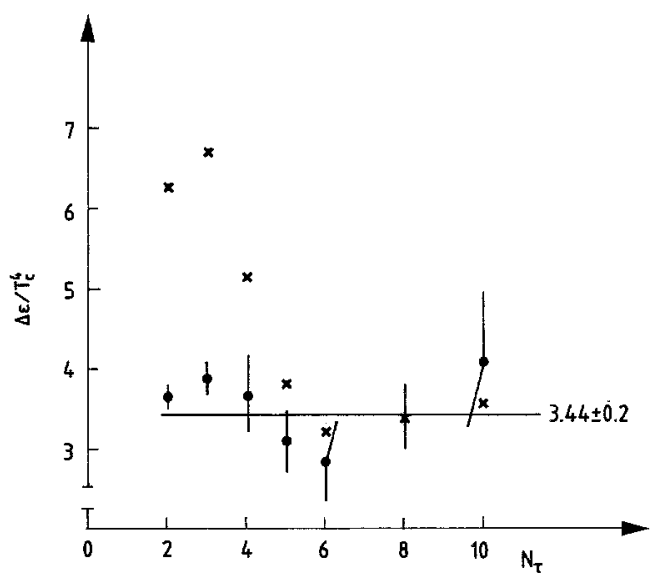

Fig. 4. Latent heat $\Delta \varepsilon / T_{c}^{4}$ for $S U(3)$ pure gauge theory versus $N_{\tau}$. Data are taken from $[6,33,34]$ and finite size corrections [35] have been taken into account. The crosses indicate uncorrected data

count to arrive at this conclusion. However, by now the energy density has been studied on rather larger lattices with up to $N_{\tau}=10$ sites in temporal direction $[6,33-34]$. The results obtained confirm the calculations on smaller lattices. This shows that the finite size effects are well under control. In Fig. 4 we show results for the latent heat of the first order deconfinement transition for $S U(3)$. Correcting for finite size effects [35] yields $N_{\tau}$-independent results for all $N_{\tau}$. From this we get for the latent heat

$\Delta \varepsilon / T_{c}^{4}=3.44 \pm 0.02=(0.65 \pm 0.04) \varepsilon_{S B} / T^{4}$.

Thus the energy density at $T_{c}$ is about $35 \%$ lower than the ideal gas value at the same temperature. The approach to this value from large temperatures is rather smooth. There are no indications for large nonperturbative corrections. In fact, from smaller lattices we know that the energy density is well described by the perturbative formula

$\varepsilon / T^{4}=a_{0}+a_{2} g^{2}+O\left(\left|g^{3}\right|\right)$

with $a_{0}, a_{2}$ calculated on finite lattices [36]. On infinite lattices the parameters approach the corresponding continuum formula, which for $S U(N)$ and $n_{f}$ massless fermion flavours are given by

$$
\begin{aligned}
& a_{0}=\frac{\pi^{2}}{15}\left(N^{2}-1+\frac{7}{4} N n_{f}\right) \\
& a_{2}=-\frac{1}{48}\left(N^{2}-1\right)\left(N+\frac{5}{4} n_{f}\right) .
\end{aligned}
$$

The coupling $g^{2}$ appearing in (3.3) is the temperature dependent running coupling constant

$$
g^{2}(T)=\frac{24 \pi^{2}}{\left(33-2 n_{f}\right) \ln \left(x T / A_{M S}\right)} .
$$


Here $x$ is a still unknown parameter which could in principle be determined in perturbation theory by performing a $O\left(\mathrm{~g}^{4}\right)$ calculation for the energy density [37]. We can extract $x$ from the Monte Carlo data for the latent heat by assuming that this can be described by (3.3) at $T_{c}$. From (3.2) and (3.3) we find then

$n_{f}=0: \quad x=3.9_{-1.6}^{+2}$.

The strong coupling constant at $T_{c}$ thus becomes

$\alpha_{s}\left(T_{c}\right)=\frac{g^{2}\left(T_{c}\right)}{4 \pi}=0.29_{-0.05}^{+0.11}$.

In general, we find that the energy density of the $S U$ (3) gluon gas above $T_{c}$ is well described by an ideal gas with $O\left(\mathrm{~g}^{2}\right)$ perturbative corrections. In Fig. 5, we show $\varepsilon / T^{4}$ on a large temperature interval.

The situation is, however, quite different for the pressure. Close to $T_{c}$ strong deviations from the ideal gas relation, $\varepsilon=3 p$, are visible. This is true for $S U(2)$ [32] and $S U(3)$ [38] pure gauge theories as well as in the presence of dynamical fermions. In Fig. 6, we show results for $S U(3)$ with $n_{f}=2$ flavours of quarks taken from [10]. These large deviations from the ideal gas value can hardly be explained by perturbation theory. In fact, from an analysis of $S U(2)$ data Källman found that this non-perturbative effect can be parametrized with a term proportional to $T$ [39]

$P=\frac{\pi^{2}}{45}\left(N^{2}-1\right) T^{4}-c T$

with $c \simeq 0.2\left(N^{2}-1\right) T_{c}^{3}$. Indeed this is also in agreement with the $S U$ (3) data [38] although the accuracy of the data is still quite bad. A more detailed analysis of the equation of state for pure $S U(3)$ gluon matter is certainly needed. In particular, it should be worked out whether in the presence of dynamical fermions

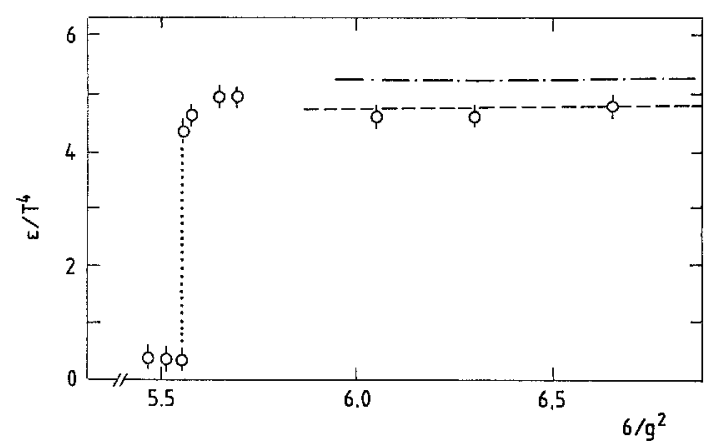

Fig. 5. $\varepsilon / T^{4}$ versus $\beta=6 / g^{2}$ for $S U(3)$ on a $10^{3} \times 3$ lattices. Also shown is the lowest order $(-.-)$ and $O\left(\mathrm{~g}^{2}\right)(--)$ weak coupling perturbative result [36]

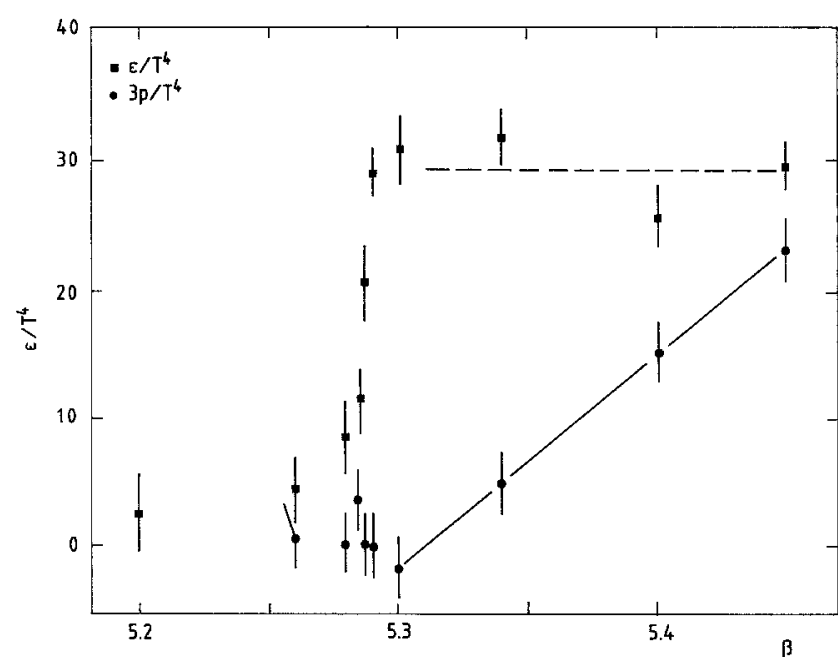

Fig. 6. $\varepsilon / T^{4}$ (squares) and $3 p / T^{4}$ (dots) versus $\beta$ for $S U(3), n_{f}=2$, $m / T=0.1$. Data are taken from [10]. Lines are drawn to guide the eye

the non-perturbative term in the pressure is also proportional to $T$.

We note that due to the relation of $\varepsilon$ and $p$ to the entropy

$S=\frac{1}{T}(\varepsilon+P)$

these results predict a substantial decrease in the entropy of the quark gluon plasma compared to an ideal gas close to $T_{c}$. The origin of the quasi ideal gas behaviour and the non-perturbative nature of the pressure has been addressed to the presence of massive plasma excitations $[15,38,40]$ which besides of a massless phonon mode [41] are expected to contribute to energy density as well as pressure in the plasma phase. The basic idea is the observation that in the plasma phase, heavy colour singlet excitations will contribute dominantly in the low momentum regime to the thermodynamic potential, while for large momenta a free massless gluon gas gives the dominant contribution. In the simplest version we may think of a single mass scale $m_{c}$ which defines the relevant momentum cut-off for these two different regimes. The thermodynamic potential reads then

$\ln Z=\alpha V f\left(T, m_{c}\right)$

with $\alpha$ denoting the effective number of degrees of freedom and

$$
\begin{aligned}
f\left(T, m_{c}\right) & =\frac{-1}{2 \pi^{2}} \int_{m_{c}}^{\infty} d k k^{2} \ln \left(1-e^{-k / T}\right) \\
& -\frac{1}{2 \pi^{2}} \int_{0}^{m_{c}} d k k^{2} \ln \left(1-e^{-\frac{1}{T} V k^{2}+m_{c}^{2}}\right) .
\end{aligned}
$$


Defining

$h\left(T, m_{c}\right)=-\frac{T^{3}}{2 \pi^{2}} \int_{0}^{m_{c} / T} d x x^{2} \ln \frac{1-e^{-V \overline{x^{2}+\left(m_{c} / T\right)^{2}}}}{1-e^{-x}}$

$a_{S B}=-\frac{1}{2 \pi^{2}} \int_{0}^{\infty} d x x^{2} \ln \left(1-e^{-x}\right)$

we can separate the ideal gas contribution in (3.9) which now reads

$\ln Z=\alpha V T^{3} a_{S B}-\alpha V h\left(T, m_{c}\right)$.

For energy density and pressure we find

$P=\alpha a_{S B} T^{4}-\alpha T h\left(T, m_{c}\right)$

$\varepsilon=3 \alpha a_{S B} T^{4}-\alpha T^{2} \frac{\partial}{\partial T} h\left(T, m_{c}\right)$.

The first terms in $(3.13 \mathrm{a} / \mathrm{b})$ give the ideal gas behaviour while the function $h\left(T, m_{c}\right)$ parametrizes the deviations. We see that indeed we can describe the $\mathrm{MC}$ data for pressure and energy density with this ansatz, if $h\left(T, m_{c}\right)$ turns out to be only weakly temperature dependent. This is the case for a temperature independent cut-off $m_{c}$. Comparing with the MC data for $S U(2)$ one finds

$m_{\mathrm{c}} \simeq 2 T_{\mathrm{c}}$.

This shows that $1 / m_{c}$ is compatible with standard zero temperature confinement scales of $\sim 1 \mathrm{fm}$, it does not seem to be related to high temperature screening lengths which would favour a linear increase of $m_{c}$ with $T[38]$.

Up to now we have discussed the thermodynamics in the pure gauge sector. Does the situation change in the presence of fermions? Looking at Figs. 5 and 6 the overall behaviour seems to be similar. However, a closer analysis of the energy density in the presence of dynamical fermions shows that its high temperature limit is far away from the expected ideal gas result. Also a comparison with finite lattice perturbation theory is still unsatisfactory [36]. This is shown in Fig. 7 where we compare the Monte Carlo data for $S U(3)$ with two light flavours [10] with the perturbative results of [36]. We note that the $O\left(\mathrm{~g}^{2}\right)$ corrections on this small lattice have the opposite sign compared to the continuum perturbative result, $(3.4 \mathrm{~b})$. Thus the MC data follow the trend of finite lattice perturbation theory. However, these finite size effects seem to be so large that we have to wait for $\mathrm{MC}$ results on larger lattices to discuss the equation of state with dynamical fermions on the basis of MC data.

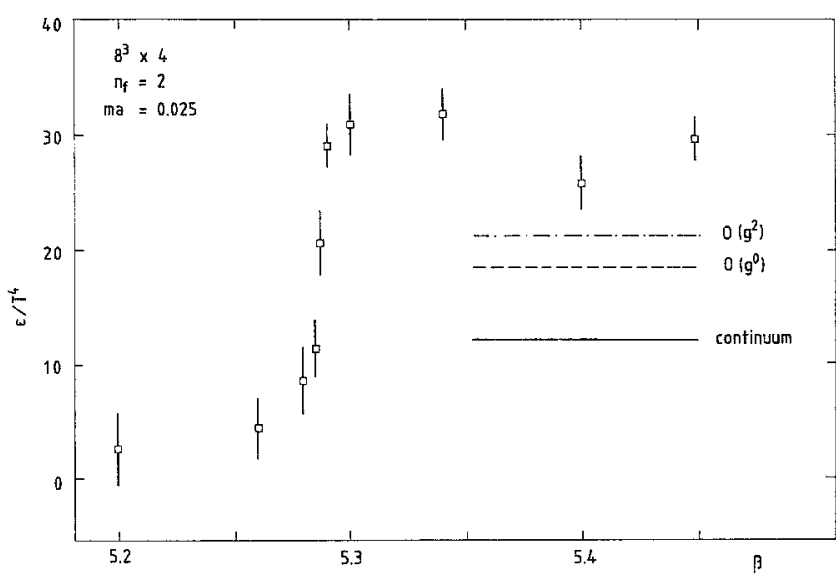

Fig. 7. $\varepsilon / T^{4}$ versus $\beta$. Same data as in Fig. 6. Also shown is the continuum Stefan-Boltzman limit (-) and finite lattice perturbative results to lowest order (---) and $O\left(g^{2}\right)(-.-$.$) from [36]$

\section{Screening lengths}

We have seen that non-perturbative effects show up in the equation of state for the quark-gluon plasma. Close to $T_{c}$ collective plasma excitations give important contributions to the pressure.

Collective modes, leading to an electric and magnetic screening length [42] have been discussed in the past as a solution for the well-known infra-red problem of the finite temperature perturbative expansion [43] of the thermodynamical potential. It is expected that the gluon propagator develops a nonperturbative electric $\left(m_{D}\right)$ and magnetic $\left(m_{M}\right)$ screening mass of $O(g)$ and $O\left(g^{2}\right)$, respectively, $m_{D}(T) \sim g(T) T$, $m_{M}(T) \sim g^{2}(T) T$. Lowest order perturbation theory yields the gauge invariant result

$m_{D}^{2}(T)=\left(\frac{N}{3}+\frac{n_{f}}{6}\right) g^{2}(T) T^{2}$

for the Debye mass $\left(m_{D}\right)$. However beyond this, little is known about these screening masses from perturbation theory. The magnitude of the electric (Debye) screening mass became of interest during the last year due to its importance in the discussion of the suppression of heavy flavour resonances as a signal of plasma formation [17]. Assuming that the QCD confinement potential is replaced by a Debye screened Coulomb potential above $T_{c}$,

$V(r, T)=\left\{\begin{array}{cc}\frac{\alpha(T)}{r}+\sigma(T) r, & T<T_{c} \\ \frac{\alpha(T)}{r} e^{-m_{D}(T) r}+c(T), & T \geqq T_{c}\end{array}\right.$

Matsui and Satz [17] derive a condition that has to be fulfilled in order to forbid the formation of bound 
states for quarks of mass $m_{q}$ in the above potential

$m_{D}(T)>1.68 m_{q} \alpha(T)$.

We thus realize the importance of a quantitative determination of $m_{D}(T)$. On the lattice, information on the electric screening length can be obtained from correlations of static heavy quarks, i.e., Polyakov loop correlation functions

$e^{-F(\boldsymbol{r}, T) / T}=\left\langle\operatorname{Tr} L_{0} \operatorname{Tr} L_{\mathbf{x}}^{+}\right\rangle$

with $r=|\mathbf{x}|, L_{\mathbf{x}}=\prod_{x_{0}=1}^{N_{\tau}} U_{\left(\mathbf{x}_{0}, x\right), 0}$ and $F(r, T)$ denoting

the change of free energy due to the presence of a static quark-antiquark pair. At large distances this correlation function gives

$\lim _{r \rightarrow \infty} e^{-F(r, T) / T}=\left\langle\operatorname{Tr} L_{0}\right\rangle^{2}$.

Assuming that for large distances the free energy behaves like

$F(r, t)=f(r, T) e^{-\mu(T) r}+c(T)$

with $c(T)=-T \ln \left\langle\operatorname{Tr} L_{0}\right\rangle^{2}$ and $\mu(T)$ the screening mass for this correlation function we can extract $\mu(T)$ from $\mathrm{MC}$ measurements of the correlation functions, (4.4). Results for $S U(2)$ [14] and $S U(3)$ [13] are shown in Figs. 8 and 9. From Fig. 8 we see that for $S U(2) \mu(T)=1 / \xi$ is a rapidly increasing function of $T$. In fact, it seems to grow faster than $T, \mu(T) / T$ starts growing close to $T_{c}$ reaching a value of about 4 for large $T$. For $S U(3) \mu(T) / T$ is roughly constant

$\mu(T) / T \simeq 2-3$ for $T_{c} \lesssim T \lesssim 2 T_{c}$.

Simulations with dynamical fermions seem to give similar results [44]. They also have the tendency to give values for $\mu(T) / T$ which increase with temperature rather than decrease as one would expect on the basis of the perturbative relation (4.1). This points to various problems with the determination of the Debye screening length which have not been clarified up to now. The relation of the screening mass $\mu(T)$ defined through (4.4)-(4.6) to the Debye mass, being defined as the screening mass in the heavy quark colour singlet potential, is not straightforward. In fact $\mu(T)$ is related to the colour averaged potential (free energy) [45], at least in lowest order perturbation theory, which leads to the conclusion that in the high temperature limit $\mu(T)=2 m_{D}(T)$. This may explain why $\mu(T) / T$ seem to be an increasing function of $T$. A further problem is that the screening length should be extracted from the correlation functions at large distances which have not been reached in present $\mathrm{MC}$

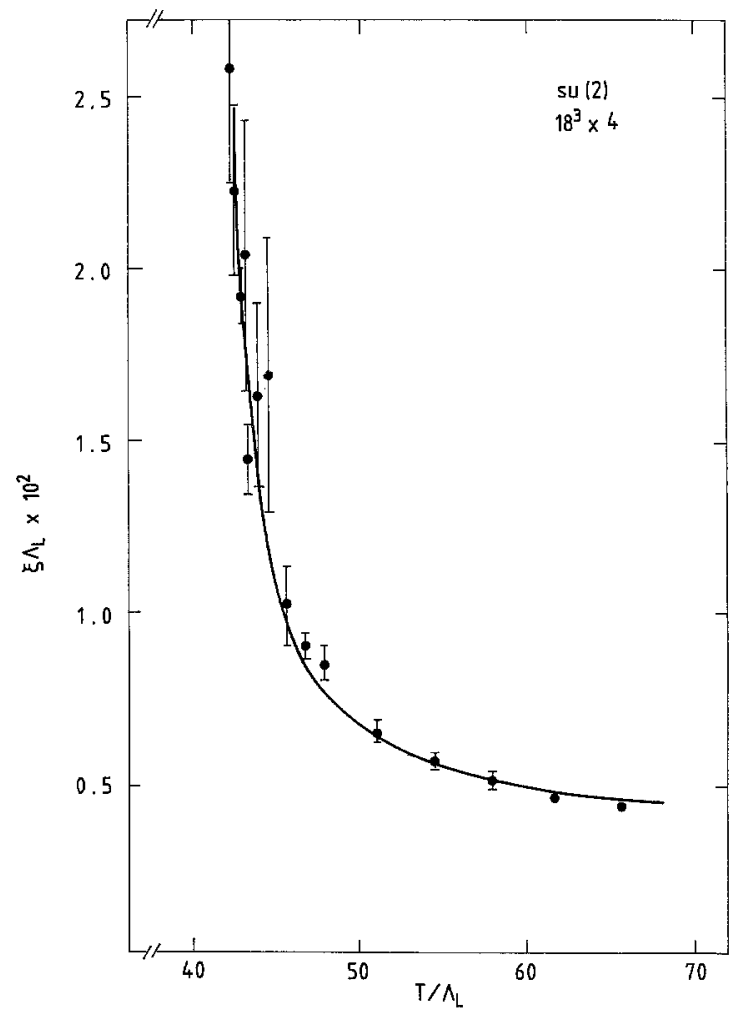

Fig. 8. Debye screening lengths $\xi \equiv 1 / m_{D}$ in units of $100 A_{L}$ versus $T / A_{L}$ for $S U(2)$ gauge theory on a $18^{3} \times 4$ lattice. Data are taken from Ref. [14]

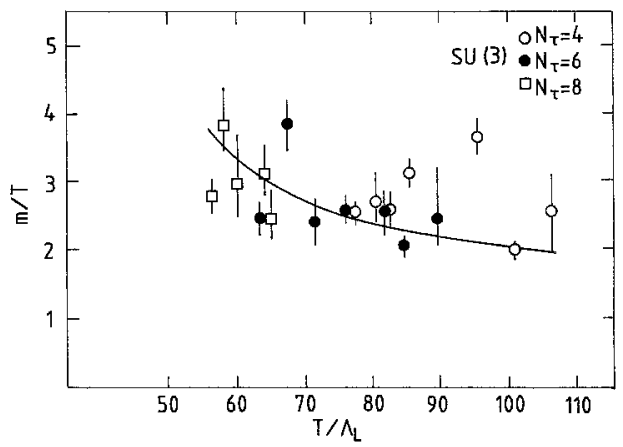

Fig. 9. Debye mass in units of $T$ as a function of $T / A_{L}$ for pure $S U$ (3) gauge theory. The figure has been taken from [13]

simulations. At short distances the heavy quark potential does not have to be of the simple Debye screened Coulomb form [46]. The determination of $\mu(T)$ from $\mathrm{MC}$ data is thus very sensitive to the specific ansatz for $f(r, T)(4.6)$ used in the fits to the data.

Also finite lattice effects that lead to a distortion of the Coulomb potential have not been taken into account in present fits. The determination of the Debye screening mass should, therefore, be considered as being in an exploratory stage. Further work is necessary to get a quantitative understanding of the heavy quark potential in the plasma phase. 


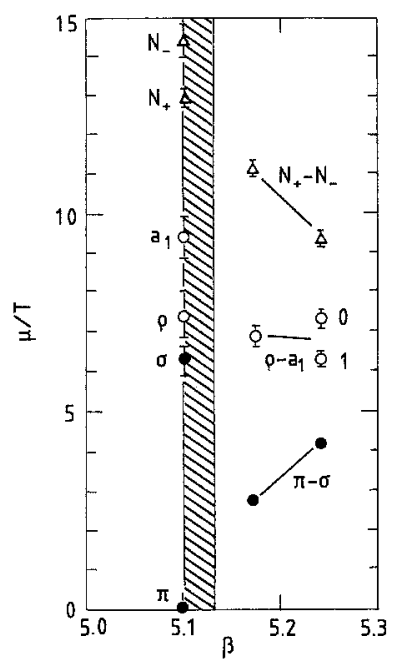

Fig. 10. Various hadronic screening masses in units of $T$ versus coupling $\beta \equiv 6 / g^{2}$ for $S U(3)$ with four light flavours. The dashed region indicates the transition region to the chiral symmetric phase with massive pionic mode $(\pi)$. The scalar/pseudoscalar $(\pi-\sigma)$, vector/pseudovector $\left(\rho-a_{1}\right)$ and nucleon parity doublet $\left(N_{+}-N_{-}\right)$ seem to have degenerate screening masses. The figure has been taken from [16]

Let us finally mention a rather interesting analysis of hadronic screening lengths in the quark-gluon plasma performed recently by C. DeTar and J.B. Kogut [16]. They study spatial correlation functions of operators with hadronic quantum numbers, i.e., correlation functions that define ordinary hadron masses at zero temperature. Above $T_{c}$ chiral symmetry is exact in the massless limit and the full $S U\left(n_{f}\right) \times S U\left(n_{f}\right)$ flavour symmetry of QCD is restored. That should be visible in the "screening mass" spectrum for these operators. In particular, the pion is no longer a Goldstone particle and thus may be massive above $T_{c}$, in addition scalar (vector) and pseudoscalar (vector) modes should have degenerate masses above $T_{c}$ as they are related through an exact symmetry transformation. These features are borne out by their data for $S U$ (3) with four light dynamical flavours shown in Fig. 10. This clear shows the presence of hadronic modes in the quark-gluon plasma. However, these excitations are quite heavy. As discussed before, they will only contribute to the low frequency part of the thermodynamic potential. Their relevance in the thermodynamics of the plasma phase thus deserves further studies. In particular, the stability of these modes has to be analyzed.

\section{Conclusions}

Lattice simulations at finite temperature are at present in a transient stage. In the pure gauge sector they have reached a stage where reliable quantitative re- sults can be obtained. The deconfinement temperature as well as the latent heat of the $S U(3)$ deconfinement transition seem to be well established

$$
\begin{aligned}
& T_{c} / \sqrt{\sigma}=0.58 \pm 0.04 \\
& \Delta \varepsilon / T_{c}^{4}=3.44 \pm 0.02
\end{aligned}
$$

Moreover we found indications for non-perturbative features of the plasma phase in the non-perturbative behaviour of the pressure and the existence of a nonvanishing Debye screening length. The quantitative analysis of this effect, however, has to be pursued further.

Simulations with dynamical fermions just start to reach a stage where large scale simulations become possible to extract quantitative results relevant for the continuum limit. Algorithms have been developed which have small enough systematic errors so that they can be used for simulations with small quark mass on large lattices. First results are encouraging. They have established the existence of a first order chiral phase transition and indicate that $T_{c} / \Lambda_{M S}$ shows little flavour dependence. Thus the results found in the pure gauge sector seem to be a good guide for continuum physics. However, we have also seen that some observables like the energy density show large finite size effects in the presence of dynamical fermions. These effects have to be analyzed in detail before any conclusions can be drawn about the equation of state of the QCD plasma with light dynamical fermions.

Acknowledgements. I would like to thank J. Rafelski, H. Satz and L. Van Hove for helpful discussions during the time of preparation of this work.

\section{References}

1. M. Jacob, H. Satz (eds.): Quark matter formation and heavy ion collisions. Singapore: World Scientific 1982

2. See this issue of Z. Phys. C - Particles and Fields

3. I. Barbour et al.: Nucl. Phys. B 275 [FS 17] (1986) 296

4. A.D. Kennedy, J. Kuti, S. Meyer, B.J. Pendleton: Phys. Rev. Lett. 54 (1985) 87; S.A. Gottlieb et al.: Phys. Rev. Lett. 55 (1985) 1958

5. N.H. Christ, A.E. Terrano: Phys. Rev. Lett. 56 (1986) 111

6. H.-Q. Ding: Lattice gauge theory at finite temperature: a Monte Carlo study. Columbia preprint (May 1987); N.H. Christ, in: Lattice gauge theory '86. H. Satz, I. Harity, J. Potvin, (Eds.), NATO AS1 Series, Vol. B 159 New York: Plenum Press 1987

7. R. Gupta et al.: Phys. Rev. Lett. 57 (1986) 2621

8. E.V.E. Kovacs, D.K. Sinclair, J.B. Kogut: Phys. Rev. Lett. 58 (1987) 751

9. F. Karsch, J.B. Kogut, D.K. Sinclair, H.W. Wyld: Phys. Lett. 188 B (1987) 353

10. S. Gottlieb et al.: Phys. Rev. D35 (1987) 3972

11. M. Fukugita, S. Ohta, Y. Oyanagi, A. Ukawa: Phys. Rev. Lett. $58(1987) 2515$ 
12. R.V. Gavai, J. Potvin, S. Sanielevici: Phys. Rev. Lett. 58 (1987) 2519

13. T.A. DeGrand, C.E. DeTar: Phys. Rev. D 34 (1986) 2469

14. K. Kanaya, H. Satz: Phys. Rev. D34 (1986) 3193

15. E. Manousakis, J. Polonyi: Phys. Rev. Lett. 58 (1987) 847

16. C.E. DeTar, J.B. Kogut: Phys. Rev. Lett 59 (1987) 599; Phys. Rev. D36 (1987) 2828

17. T. Matsui, H. Satz: Phys. Lett. 178 B (1986) 416

18. C. Borgs, E. Seiler: Commun. Math. Phys. 91 (1983) 195

19. E.T. Tomboulis, L.G. Yaffe: Phys. Rev. Lett. 52 (1984) 2115

20. T. Celik, J. Engels, H. Satz: Phys. Lett. 125B (1983) 411; J. Kogut et al.: Phys. Rev. Lett. 50 (1983) 393

21. K.C. Bowler et al.: Nucl. Phys. B257 [FS 14] (1985) 155

22. K.C. Bowler et al.: Phys. Lett. 163 B (1985) 225 42; Ph. de Forcrand: J. Stat. Phys. 43 (1986) 1077; H.-Q. Ding: Phys. Lett. $200 \mathrm{~B}(1988) 133$

23. K.C. Bowler et al.: Nucl. Phys. B296 (1988) 732

24. S. Jacobs, M.G. Olsson, C. Suchyta III: Phys. Rev. D 33 (1986) 3338

25. T. Banks, A. Ukawa: Nucl. Phys. B 225 [FS9] (1983) 145

26. For a review and references for the intermediate quark mass regime, see: J. Cleymans, R.V. Gavai, E. Suhonen: Phys. Rep. 130 (1986) 21.7

27. J.B. Kogut, J. Polonyi, D.K. Sinclair: Phys. Rev. Lett. 55 (1985) 1475; N.A. Attig, B. Petersson, M. Wolff: Phys. Lett. 190B (1987) 143. M. Fukugita, S. Ohta, A. Ukawa: Phys. Rev. Lett. 60 (1988) 178

28. R. Pisarski, F. Wilczek: Phys. Rev. D 29 (1984) 338

29. J.B. Kogut, D.K. Sinclair: Illinois preprint ILL-(TH)-87-47 (1987)
30. A. Billoire, E. Marinari: Phys. Lett. 184 (1987) 381 ; M. Fukugita, S. Ohta, Y. Oyanagi, A. Ukawa: Phys. Lett. 191 B (1987) 164

31. J. Kogut et al, : Illinois preprint, ILL-(TH)-88-\#2, 1988; R.V. Gavai, J. Potvin, S. Sanielevici: Brookhaven preprint, BNL 40257,1987

32. J. Engels, F. Karsch, I. Montvay, H. Satz: Phys. Lett. $101 \mathrm{~B}$ (1981) 89; Nucl. Phys. 205 B [FS5] (1982) 545

33. T. Celik, J. Engels, H. Satz: Phys. Lett. 129 B (1983) 323, Z. Phys. C - Particles and Fields 22 (1984) 301

34. F. Karsch, R. Petronzio: Phys. Lett. 139 B (1984) 403

35. J. Engels, F. Karsch, H. Satz: Nucl. Phys. B205 [FS5] (1982) 239

36. U. Heller, F. Karsch: Nucl. Phys. B251 [FS13] (1985) 254; Nucl. Phys. B258 (1985) 29

37. See, for instance, the discussion in: K. Kajantie, J. Kapusta: Ann. Phys. (NY) 169 (1985) 477

38. T.A. DeGrand, C.E. DeTar: Phys. Rev. D 35 (1987) 742

39. C.G. Källman: Phys. Lett. 134B (1984) 363

40. M.I. Gorenstein, O.A. Mogilevsky: Kiev preprint ITP-87-83E (1987)

41. P. Carruthers: Phys. Rev. Lett. 50 (1983) 1179

42. For a discussion, see: D.J. Gross, R.D. Pisarski, L.G. Yaffe, Rev. Mod. Phys. 53 (1981) 43

43. A. Linde: Rep. Prog. Phys. 42 (1979) 389

44. R.V. Gavai, M. Lev, B. Petersson, H. Satz: Bielefeld preprint, BI-TP 87/23

45. S. Nadkarni: Phys. Rev. D 33 (1986) 3738; Phys. Rev. D 34 (1986) 3904

46. C. Gale, J. Kapusta: Univ. of Minnesota preprint (1987) 